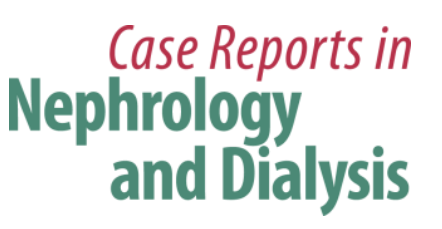

Case Rep Nephrol Dial 2019;9:1-7

DOI: 10.1159/000496465

Published online: February 8, 2019

(c) 2019 The Author(s)

Published by S. Karger AG, Basel

www.karger.com/cnd

This article is licensed under the Creative Commons Attribution-NonCommercial 4.0 International License (CC BY-NC) (http://www.karger.com/Services/OpenAccessLicense). Usage and distribution for commercial purposes requires written permission.

\title{
Progressive Renal Dysfunction due to IgG4-Related Kidney Disease Refractory to Steroid Therapy: A Case Report
}

\author{
Keiichi Wakabayashi ${ }^{a}$ Hiroyuki Yanagawa ${ }^{a}$ Yoko Hayashi $^{a}$ \\ Rumi Aoyama $^{a}$ Yoshio Shimizu $^{a, c}$ Yasuhiko Tomino ${ }^{b}$ Yusuke Suzuki ${ }^{b}$ \\ aDivision of Nephrology, Department of Internal Medicine, Juntendo Shizuoka Hospital, \\ Izunokuni, Japan; ${ }^{b}$ Division of Nephrology, Department of Internal Medicine, Juntendo \\ University Faculty of Medicine, Tokyo, Japan; 'Shizuoka Medical Research Center for \\ Disaster, Juntendo University, Izunokuni, Japan
}

\section{Keywords}

IgG4-related kidney disease · Steroid therapy · Progressive renal dysfunction

\begin{abstract}
Recently, as the number of case reports of IgG4-related kidney disease (IgG4-RKD) has increased, the histopathological features and clinical approach have been clarified. IgG4-RKD generally has a benign prognosis due to the efficacy of steroid therapy and rarely requires dialysis. Herein, we report a case of IgG4-RKD that presented with a subacute onset, advanced to end-stage kidney disease, and finally required maintenance hemodialysis despite steroid therapy. A 75-year-old man was admitted to our hospital for further evaluation of subacute renal failure. Diffuse enlargement of the kidney on computed tomography and increased urinary $\mathrm{N}$-acetyl- $\beta$-D-glucosaminidase and $\alpha 1$-microglobulin levels led us to suspect IgG4-RKD. Upon admission, the laboratory serological findings were as follows: creatinine $3.3 \mathrm{mg} / \mathrm{dL}$, urea nitrogen $46.9 \mathrm{mg} / \mathrm{dL}$, and IgG4 $235 \mathrm{mg} / \mathrm{dL}$. Urinalysis showed slight proteinuria without hematuria. Percutaneous renal needle biopsy showed diffuse infiltration of abundant lymphocytes and IgG4-positive plasma cells and storiform fibrosis, which is specific to IgG4-RKD, in the interstitium on light microscopy. Slight linear deposition of $\mathrm{C} 3$ was also observed in the tubules
\end{abstract}




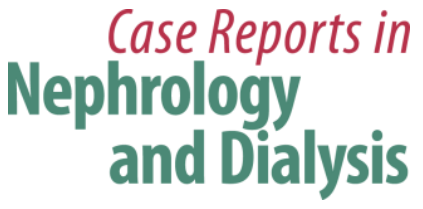

Case Rep Nephrol Dial 2019;9:1-7

DOI: $10.1159 / 000496465$

(c) 2019 The Author(s). Published by S. Karger AG, Basel www.karger.com/cnd

Wakabayashi et al.: IgG4-Related Kidney Disease Refractory to Steroid Therapy

on immunofluorescence microscopy, with no electron-dense deposits. He was definitively diagnosed as having $\lg$ G4-RKD and started on prednisolone $0.6 \mathrm{mg} / \mathrm{kg} /$ day. However, the renal insufficiency continued to progress and hemodialysis was necessary. As the prednisolone was tapered, renal function did not improve and maintenance hemodialysis was started. In conclusion, this case indicates that the prognosis of IgG4-RKD is not necessarily benign and that further studies involving more patients are needed.

(C) 2019 The Author(s)

Published by S. Karger AG, Basel

\section{Introduction}

IgG4-related disease (IgG4-RD) is a systemic inflammatory disorder defined by the combined presence of a characteristic histopathological appearance (i.e., abundant infiltration of plasma cells with IgG4), interstitial fibrosis in the involved organs, i.e., the pancreas, gallbladder, salivary glands, retroperitoneum, lungs, prostate and kidney, and elevated serum levels of IgG4 [1].

The most common feature of the renal involvement in IgG4-RD is tubulointerstitial nephritis with abundant IgG4-positive plasma cells and storiform fibrosis, which are findings specific to IgG4-RD, in the interstitium. However, some cases with glomerular injury, i.e., membranous glomerulonephritis, IgA nephropathy, and membranoproliferative glomerulonephritis, have also been described [2]. Consequently, while increasing levels of $\mathrm{N}$-acetyl- $\beta$-D-glucosaminidase (NAG) and $\alpha 1$-microglobulin $(\alpha 1-\mathrm{MG})$ are the main findings of IgG4-related kidney disease (IgG4-RKD) on urinalysis, slight proteinuria and microhematuria may also appear. Computed tomography (CT) is the most recommended radiographic imaging method for IgG4-RKD. However, the use of contrast medium requires careful judgment in patients with impaired renal function. Diffuse enlargement of the kidney on noncontrast CT and multiple low-density lesions on enhanced CT are the most common findings. A hypovascular solitary nodule in the kidney and diffuse thickening of the renal pelvis wall are rarely observed on CT, and it is necessary to distinguish these findings from malignant tumors [2]. Moreover, IgG4RKD an often be accompanied by autoimmune pancreatitis (AIP) and Mikulicz disease. However, several cases without these diseases have also been reported [2]. As such, the clinical course of IgG4-RKD is varied and it is sometimes difficult to diagnose.

IgG4-RKD is thought to be responsive to steroid therapy, similar to AIP [1]. Saeki et al. [3] reported that decreased renal function, hypocomplementemia, or abnormal renal radiologic findings improved rapidly 1 month after the start of steroid therapy in 18 of 19 patients with IgG4-RKD. Moreover, maintenance therapy with low-dose prednisolone is recommended to prevent relapse [1].

IgG4-RKD generally has a benign prognosis due to its responsiveness to steroid therapy and rarely requires dialysis. Recently, while the number of case reports associated with IgG4RKD has been increasing and the histopathological feature and clinical approach have been clarified, it remains controversial. Herein, we report a case of IgG4-RKD who presented with subacute onset, advanced to end-stage kidney disease, and finally required maintenance hemodialysis despite the administration of glucocorticoid therapy.

\section{Case Report}

A 75-year-old man was admitted to our hospital for further evaluation of subacute renal failure. He had no past history associated with bronchial asthma or drug administration and 


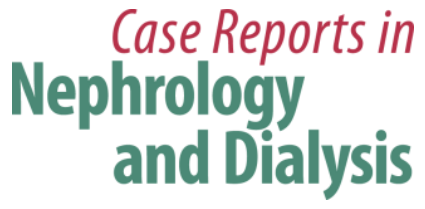

Case Rep Nephrol Dial 2019;9:1-7

DOI: $10.1159 / 000496465$

(c) 2019 The Author(s). Published by S. Karger AG, Basel www.karger.com/cnd

Wakabayashi et al.: IgG4-Related Kidney Disease Refractory to Steroid Therapy

no other medical problems, history of smoking, habitual drinking, or family history of renal disease. Upon admission, his body weight was $51.0 \mathrm{~kg}$, height was $171.0 \mathrm{~cm}$ (body mass index 17.4), temperature was $36.8^{\circ} \mathrm{C}$, and blood pressure was $125 / 74 \mathrm{~mm} \mathrm{Hg}$. The lymph nodes were not palpable, and there were no signs of leg edema. The peripheral white blood cell count was $4,600 / \mathrm{mm}^{3}$, with 3,450 neutrophils $/ \mathrm{mm}^{3}$ and 92 eosinophils $/ \mathrm{mm}^{3}$; the hematocrit was $27.1 \%$; the red blood cell count was $2,710,000 / \mathrm{mm}^{3}$, hemoglobin was $8.9 \mathrm{~g} / \mathrm{dL}$, and the platelet count was $156,000 / \mathrm{mm}^{3}$. Laboratory serological findings were as follows: total protein $7.5 \mathrm{~g} / \mathrm{dL}$, albumin $4.4 \mathrm{~g} / \mathrm{dL}$, creatinine $3.3 \mathrm{mg} / \mathrm{dL}$, urea nitrogen $46.9 \mathrm{mg} / \mathrm{dL}$, and C-reactive protein $0.6 \mathrm{mg} / \mathrm{dL}$. The serum IgG and IgG4 levels were $1,614.0$ and $235.0 \mathrm{mg} / \mathrm{dL}$, respectively. Serum protein electrophoresis showed no signs of gammopathy. Slight hypocomplementemia in C4 (C3 $83 \mathrm{mg} / \mathrm{dL}$, C4 $21.7 \mathrm{mg} / \mathrm{dL}, \mathrm{CH} 5049 \mathrm{U} / \mathrm{mL}$ ) was also observed. Antinuclear and antineutrophil cytoplasmic antibodies, antiglomerular basement membrane antibody, SS-A and SS-B antibodies, as well as cryoglobulin were all negative. Serum angiotensinconverting enzyme, soluble interleukin-2 receptor, and tumor marker levels were within normal limits. Urinalysis showed proteinuria of $0.9 \mathrm{~g}$ /day without hematuria and increasing levels of NAG (22.8 IU/L) and $\alpha 1-\mathrm{MG}(85 \mathrm{mg} / \mathrm{L})$. Whole-body CT and magnetic resonance imaging showed no pleural effusion, ascites, lymph node swelling, or enlargement of organs, except for diffuse enlargement of the kidneys (Fig. 1). The longest diameter of the kidneys was $>12 \mathrm{~cm}$. A whole-body gallium scan showed no abnormal accumulation.

Percutaneous renal needle biopsy showed diffuse infiltration of abundant lymphocytes and IgG4-positive plasma cells and storiform fibrosis, which is specific to IgG4-RKD, in the interstitium on light microscopy. There were no signs of injury of the glomeruli or basement membrane. No crescent forms were observed in glomeruli. Slight linear deposition of C3 was observed in the tubules on immunofluorescence microscopy, and no electron-dense deposits were observed (Fig. 2a, b). Immunohistochemically, IgG4-positive plasma cells were markedly observed in the interstitium ( $>10$ IgG4-positive plasma cells/high-power field), and the ratio of IgG4-positive plasma cells to IgG-positive plasma cells was >90\% (Fig. 2c, d).

The diagnosis of IgG4-RKD was reached based upon the above-described findings, and the patient was started on prednisolone at a dose of $0.6 \mathrm{mg} / \mathrm{kg} /$ day $(30 \mathrm{mg} /$ day). Serum IgG4 and creatinine levels and urinary $\alpha 1-\mathrm{MG}$ level improved temporarily; however, renal insufficiency continued to advance as the prednisolone was tapered. It became necessary to decrease the dose of the steroid after the onset of severe steroid psychosis. He lost his appetite and became malnourished, and we abandoned the additional therapy because of the risk of infection. The renal function did not improve and maintenance dialysis was initiated. Serum creatinine as well as urinary NAG and $\alpha 1-\mathrm{MG}$ levels became exacerbated as the prednisolone was tapered. However, the serum IgG4 level continued to decline. Moreover, the diffuse enlargement of kidneys, which was detected by CT, did not improve from the initiation of steroid therapy to the initiation of hemodialysis.

\section{Discussion and Conclusion}

Herein, we report a rare case of IgG4-RKD, which was limited to the kidneys and exhibited severe tubulointerstitial nephritis and required maintenance hemodialysis despite steroid therapy. This case exhibited increasing serum levels of IgG4 and a slight decrease in complement, diffuse enlargement of the kidneys on CT, storiform fibrosis and diffuse infiltration of abundant lymphocytes, and IgG4-positive plasma cells in the interstitium, in the absence of glomerulonephritis. While serum IgG4 levels tended to decrease after steroid therapy, serum 


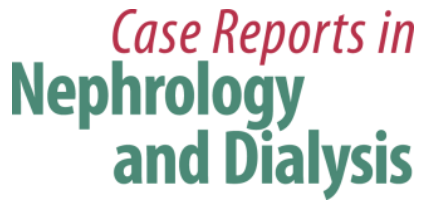

Case Rep Nephrol Dial 2019;9:1-7

DOI: 10.1159/000496465

(c) 2019 The Author(s). Published by S. Karger AG, Basel www.karger.com/cnd

Wakabayashi et al.: IgG4-Related Kidney Disease Refractory to Steroid Therapy

levels of creatinine and markers of tubular damage became exacerbated and the kidney enlargement persisted. There have been a few reports describing cases of IgG4-RKD that required maintenance hemodialysis. Saida et al. [4] reported a Japanese case of IgG4-RKD who required maintenance dialysis despite steroid therapy. In that report, IgG4-RKD developed after withdrawal of steroid therapy for AIP. The authors concluded that the reasons for the relapse were the rapid discontinuation of steroid without maintenance therapy, the high serum creatinine level at onset, and the coexistence of membranous nephropathy.

In general, IgG4-RKD has a benign prognosis due to its responsiveness to steroid therapy, similar to AIP and Mikulicz disease [5-7]. According to the therapeutic plan for AIP in Japan, it is recommended to initiate treatment with prednisolone at $0.6 \mathrm{mg} / \mathrm{kg} /$ day and to taper the dose every several weeks to $2.5-10 \mathrm{mg} /$ day as a maintenance dose. However, no consensus exists on the term for maintenance therapy. As with the recommended treatment course, prednisolone was initiated at $0.6 \mathrm{mg} / \mathrm{kg} /$ day and tapered every 4 weeks in the present case; however, this approach was not effective. Moreover, the prednisolone had to be discontinued rapidly due to the development of uremia during the tapering process.

Saeki et al. [5] reported that early treatment for IgG4-RKD is important because steroid therapy had elicited rapid, but not complete, improvement of renal function in patients with an estimated glomerular filtration rate $<60 \mathrm{~mL} / \mathrm{min}$ before therapy. The estimated glomerular filtration rate in the present case was $12.6 \mathrm{~mL} / \mathrm{min}$, which could be one of the reasons that the steroid therapy was insufficient. Kawano et al. [2] reported 41 cases of IgG4-RKD and described their characteristics. In that report, serum IgG levels were $>1,800 \mathrm{mg} / \mathrm{dL}$ in more than $90 \%$ and $>3,000 \mathrm{mg} / \mathrm{dL}$ in more than $50 \%$ of all patients. Moreover, the authors reported that only 2 cases exhibited IgG4-RD limited to the kidney and that it was common for IgG4-RD to develop simultaneously at three to four organs. In the present case, the serum IgG level was within normal limits and increased slowly, which could have delayed disease detection.

Mizushima et al. [8] reported that the findings of kidney enlargement on CT improved after steroid therapy. In this case, steroid therapy was ineffective and the enlargement of the kidney did not improve after the steroid therapy. Consequently, there was the potential to change the treatment to intravenous steroid pulse therapy or to increase the oral dose. Aoki et al. [9] reported a case of Mikulicz disease that was complicated by severe interstitial nephritis associated with IgG4 and successfully treated by methylprednisolone semipulse therapy. Moreover, some reports have shown that steroid pulse therapy is effective for the prevention of relapse in AIP patients [10] and that steroid pulse therapy is effective in reducing the maintenance dose in biliary stenosis caused by AIP [11].

With the exception of steroids, immunosuppressive agents are rarely used because steroids exhibit good efficacy against IgG4-RKD; thus, the efficacy and safety of immunosuppressives have not yet been established. However, there have been several reports using immunosuppressive agents in some refractory cases, recurrent cases, and a case of IgG4-RKD complicated by membranous glomerulonephritis. McMahon et al. [12] described the efficacy of rituximab as a steroid-sparing option for refractory IgG4-tubulointerstitial nephritis. Alexander et al. [13] stated that membranous glomerulonephritis should be included in the spectrum of IgG4-RD and should be suspected in proteinuric IgG4-RD patients. They further reported that mycophenolate mofetil and cyclophosphamide were effective in such patients. Moreover, several agents such as azathioprine, mycophenolate mofetil, and methotrexate have been attempted as steroid-sparing agents or maintenance drugs in some reports, but their effects were controversial because there were no clinical trials [11].

In conclusion, the clinical course of IgG4-RKD varies widely and it can sometimes be difficult to diagnosis. In particular, there are no reports on a case of rapidly progressive IgG4- 
RKD. Intravenous steroid pulse therapy and an increasing dose of oral steroid as well as other immunosuppressive agents should be considered in cases of rapidly progressing renal failure and pathologically severe cases, as the present case, as well as steroid-refractory and recurrent cases. This case indicates that the prognosis of IgG4-RKD is not necessarily benign and that further studies involving more patients are needed.

\section{Acknowledgment}

The authors are indebted to the nephrologists and patients at Juntendo Shizuoka Hospital for their collaboration in this study.

\section{Statement of Ethics}

The authors have no ethical conflicts to declare.

\section{Disclosure Statement}

The authors declare no conflict of interest. No funding was received for this case report.

\section{References}

1 Stone JH, Zen Y, Deshpande V. IgG4-related disease. N Engl J Med. 2012 Feb;366(6):539-51.

2 Kawano M, Saeki T, Nakashima H, Nishi S, Yamaguchi Y, Hisano S, et al. Proposal for diagnostic criteria for IgG4-related kidney disease. Clin Exp Nephrol. 2011 Oct;15(5):615-26.

3 Saeki T, Nishi S, Imai N, Ito T, Yamazaki H, Kawano M, et al. Clinicopathological characteristics of patients with IgG4-related tubulointerstitial nephritis. Kidney Int. 2010 Nov;78(10):1016-23.

4 Saida Y, Homma N, Hama H, Ueno M, Imai N, Nishi S, et al. [Case of IgG4-related tubulointerstitial nephritis showing the progression of renal dysfunction after a cure for autoimmune pancreatitis]. Nihon Jinzo Gakkai Shi. 2010;52(1):73-9.

5 Saeki T, Kawano M, Mizushima I, Yamamoto M, Wada Y, Nakashima H, et al. The clinical course of patients with IgG4-related kidney disease. Kidney Int. 2013 Oct;84(4):826-33.

6 Raissian Y, Nasr SH, Larsen CP, Colvin RB, Smyrk TC, Takahashi N, et al. Diagnosis of IgG4-related tubulointerstitial nephritis. J Am Soc Nephrol. 2011 Jul;22(7):1343-52.

7 Deshpande V, Zen Y, Chan JK, Yi EE, Sato Y, Yoshino T, et al. Consensus statement on the pathology of IgG4related disease. Mod Pathol. 2012 Sep;25(9):1181-92.

8 Mizushima I, Yamada K, Fujii H, Inoue D, Umehara H, Yamagishi M, et al. Clinical and histological changes associated with corticosteroid therapy in IgG4-related tubulointerstitial nephritis. Mod Rheumatol. 2012 Nov;22(6):859-70.

9 Aoki A, Sato K, Itabashi M, Takei T, Yoshida T, Arai J, et al. A case of Mikulicz's disease complicated with severe interstitial nephritis associated with IgG4. Clin Exp Nephrol. 2009 Aug;13(4):367-72.

10 Sugimoto M, Takagi T, Suzuki R, Konno N, Watanabe K, Nakamura J, et al. Efficacy of Steroid Pulse Therapy for Autoimmune Pancreatitis Type 1: A Retrospective Study. PLoS One. 2015 Sep;10(9):e0138604.

11 Matsushita M, Yamashina M, Ikeura T, Shimatani M, Uchida K, Takaoka M, et al. Effective steroid pulse therapy for the biliary stenosis caused by autoimmune pancreatitis. Am J Gastroenterol. 2007 Jan;102(1): 220-1.

12 McMahon BA, Novick T, Scheel PJ, Bagnasco S, Atta MG. Rituximab for the Treatment of IgG4-Related Tubulointerstitial Nephritis: Case Report and Review of the Literature. Medicine (Baltimore). 2015 Aug; 94(32):e1366.

13 Alexander MP, Larsen CP, Gibson IW, Nasr SH, Sethi S, Fidler ME, et al. Membranous glomerulonephritis is a manifestation of IgG4-related disease. Kidney Int. 2013 Mar;83(3):455-62. 


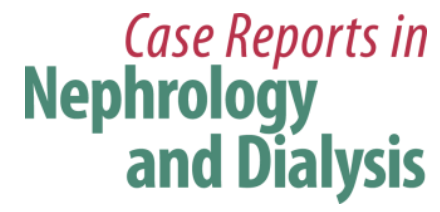

Case Rep Nephrol Dial 2019;9:1-7

DOI: $10.1159 / 000496465$

(c) 2019 The Author(s). Published by S. Karger AG, Basel www.karger.com/cnd

Wakabayashi et al.: IgG4-Related Kidney Disease Refractory to Steroid Therapy

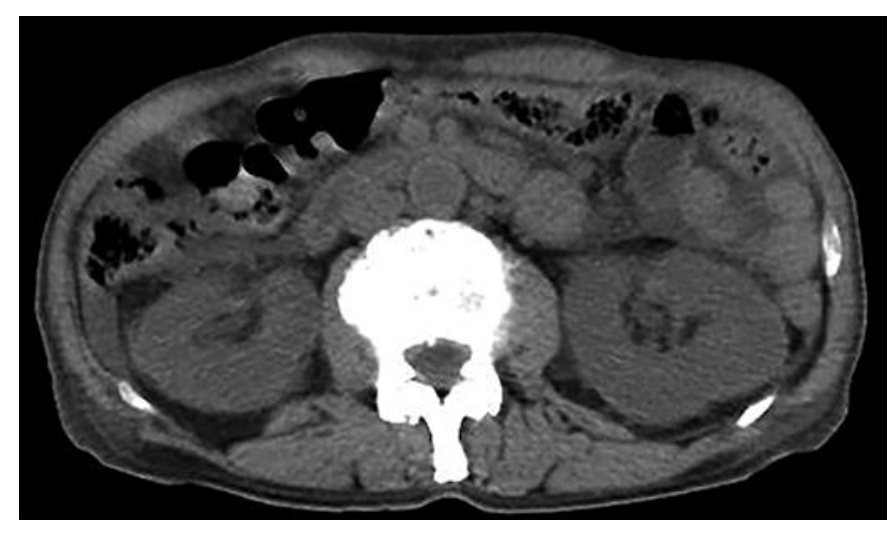

Fig. 1. Computed tomography kidney findings at onset. Diffuse enlargement of the kidneys was observed. The longest diameter of the kidneys was $>12 \mathrm{~cm}$. 


\section{Case Reports in Nephrology and Dialysis}
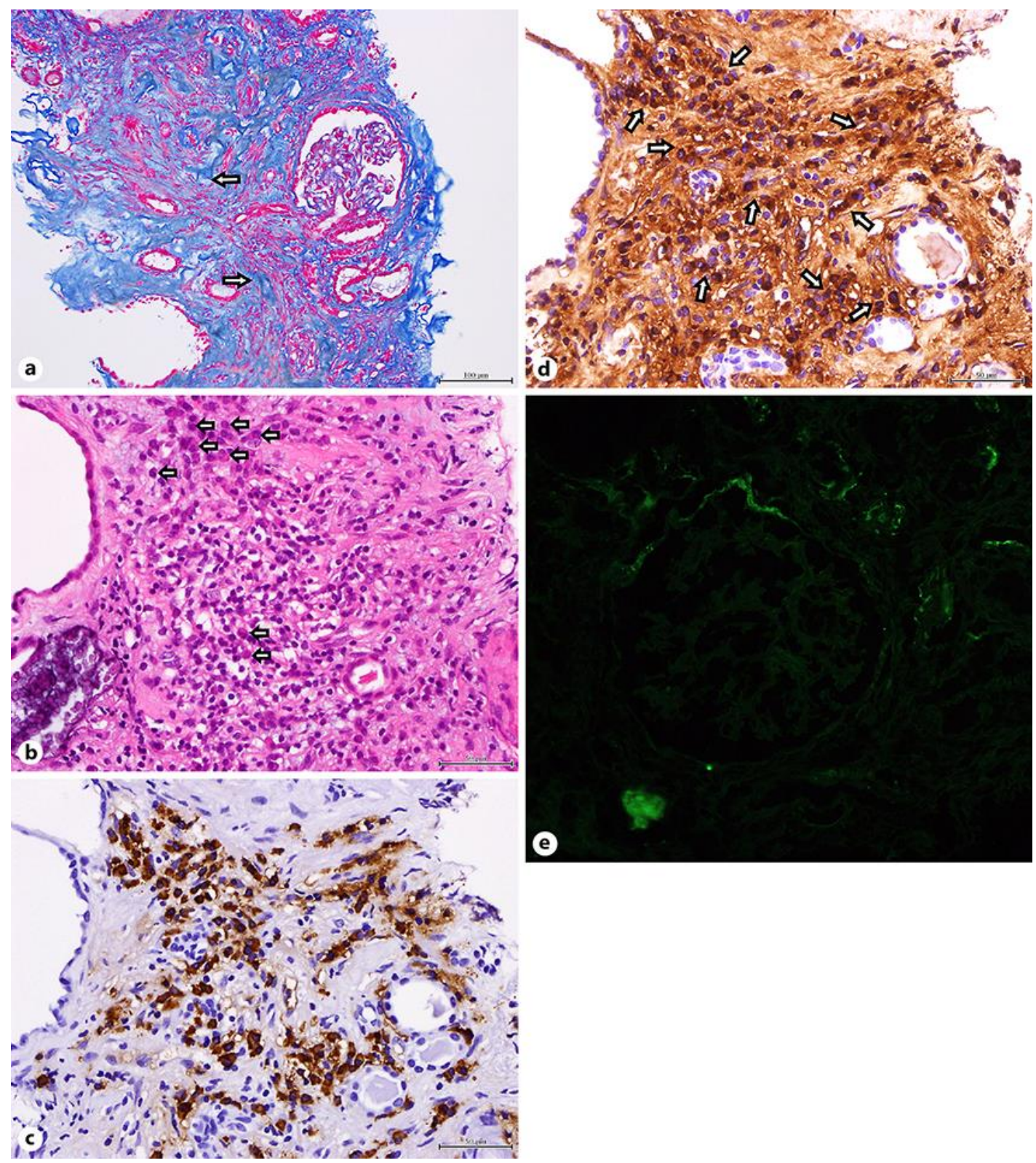

Fig. 2. Renal biopsy findings. a Normal glomeruli and storiform fibrosis, which is specific to IgG4-related kidney disease, were observed in the interstitium on light microscopy (Azan staining, $\times 100$ ). $\mathbf{b}$ Diffuse infiltration of abundant mononuclear cells and plasma cells (marked by arrows) was observed in the interstitium (hematoxylin and eosin staining, $\times 200$ ). c, $\mathbf{d}$ The immunohistochemical findings of IgG4 (c) and IgG (d) are shown. IgG4-positive plasma cells were markedly observed in the interstitium ( $>10$ IgG4-positive plasma cells/high-power field), and the ratio between IgG4-positive and IgG-positive plasma cells (marked by arrows) was $>90 \%$. e Slight linear deposition of C3 was observed in the tubules on immunofluorescence microscopy. 